\section{Intraoperative testing of opening and closing pressure predicts risk of low intraocular pressure after Ahmed glaucoma valve implantation}

F Bochmann, A Kipfer, J Tarantino, C Kaufmann, $\mathrm{L}$ Bachmann and $\mathrm{M}$ Thiel
Department of

Ophthalmology, Cantonal Hospital of Lucerne, Lucerne 16 , Switzerland

Correspondence: F Bochmann, Department of Ophthalmology, Cantonal Hospital of Lucerne, 6000 Lucerne 16, Switzerland

Tel: + 41 (0) 4120534 16 Fax: + 41 (0) 412053406 E-Mail: frank.bochmann@ luks.ch

Received: 10 November 2013

Accepted in revised form: 19 June 2014 Published online: 25 July 2014

\begin{abstract}
Purpose The aim of this study was to assess whether intraoperative testing of silicone Ahmed glaucoma valves (AGVs) would identify valves with an increased risk of low postoperative intraocular pressure (IOP).

Methods In 30 consecutive cases of glaucoma surgery with AGV implantation, after priming the $\mathrm{AGV}$, we intraoperatively measured the opening pressure $A$, closing pressure $B$, and re-opening pressure $C$ using the active infusion pump of a phakomachine. IOP was checked postoperatively on the same day. Low IOP was defined as $<5 \mathrm{~mm} \mathrm{Hg}$. Intraoperatively measured pressure characteristics of the valve function were analysed for their ability to predict postoperative IOP outcomes.

Results Opening A, closing B, and reopening $\mathrm{C}$ pressures (mean, (SD)) were 18.4 (5.1), 8.3 (4.7), and $11.7(4.8) \mathrm{mm} \mathrm{Hg}$, respectively. Ten patients $(33.3 \%)$ had low IOP. An opening pressure of $\leq 18 \mathrm{~mm} \mathrm{Hg}$ predicted low postoperative IOP with a sensitivity (10/10) of $100 \%$ (95\% CI, $69.2-100)$ and a specificity (13/20) of $65.0 \%(95 \% \mathrm{CI}$, 40.8-84.6).

Conclusions AGVs have a high variability of opening, closing, and re-opening pressures. An opening pressure of $\leq 18 \mathrm{~mm} \mathrm{Hg}$, a closing pressure of $\leq 10 \mathrm{~mm} \mathrm{Hg}$, or a re-opening pressure of $\leq \mathbf{1 1} \mathrm{mm} \mathrm{Hg}$ identified all patients with low postoperative IOP. Eye (2014) 28, 1184-1189; doi:10.1038/eye.2014.168; published online 25 July 2014
\end{abstract}

Introduction

The FP 7 silicone Ahmed glaucoma valve (AGV) (New World Medical, Rancho Cucamonga, CA, USA) is an aqueous drainage device that is increasingly used to lower intraocular pressure (IOP) in refractory glaucoma. The principle of the method is that the aqueous humour is drained through a tube to a reservoir around an end plate, from which it is reabsorbed by the surrounding tissue capsule. One unique and attractive feature of the AGV is its valve-like mechanism to prevent over-filtration and hypotony in the early postoperative phase when the capsule has not yet built up around the end plate. ${ }^{1}$ The valve is located between tube and end plate. If the pressure in the tube exceeds a certain value, two elastic membranes of the valve open and the pressure drops. Once the pressure in the tube falls below a certain value, the membranes reappose, thereby closing the valve. This mechanism is designed to ensure a minimal postoperative IOP of $8 \mathrm{~mm} \mathrm{Hg}$ (personal communication with $\mathrm{Mr}$ Ahmed ESCRS, September 2008).

Despite the outflow restriction, postoperative hypotony and related complications, such as choroidal effusion or shallow anterior chamber, are regularly reported. ${ }^{2,3}$ In vitro studies have shown a high variability of the opening and closing pressures needed to activate or deactivate the AGV, which may explain cases of hypotony after AGV implantation. ${ }^{4}$

The present study aimed to assess whether intraoperative measurement of the opening and 
closing pressure can be used to identify individual valves with an increased risk of over- or underfiltration.

\section{Materials and methods}

We performed a single-centre study of 30 consecutive cases of glaucoma surgery with implantation of an AGV between February 2011 and April 2012. The AGV implantation was not combined with any other procedure, and no viscoelastic substances were used. All surgeries were performed by the same surgeon (FB). The study was approved by the local ethics committee and each patient gave informed consent. We certify that all applicable institutional and governmental regulations concerning the ethical use of human volunteers were followed during this research.

\section{Surgical procedure}

The AGV was primed as required by the manufacturer, by gently flushing balanced salt solution through the tube and valve prior to implantation. Immediately after the priming procedure and just prior to implantation, we intraoperatively measured the opening and closing pressures of the specific AGV device to be implanted. This measurement was performed by connecting the cannula with the attached tube of the AGV to an infusion bottle of our phaco-vitrectomy machine (Oertli OS3, Oertli Instrumente AG, Berneck, Switzerland). To equilibrate the system, the bottle height had been adjusted previously to the height of the instrument table so that no flow was observed in the tubing in either direction. Then the tube was connected to the infusion bottle, and the air pressure in the infusion bottle was increased by $1-\mathrm{mm} \mathrm{Hg}$ steps using the air infusion pump of the phako-vitrectomy machine. At each pressure step, the system was allowed to equilibrate during a 5-s pause. The pressure was increased until detection of flow through the valve of the AGV, indicating AGV activation (opening pressure A). At this point, the pressure was reduced in the same stepwise manner until the flow stopped (closing pressure B). Next, the pressure was increased again until flow reappeared (re-opening pressure C). Finally, the AGV was implanted using a standardised procedure. The surgery was performed under general or parabulbar anaesthesia. All AGVs were implanted in the supratemporal quadrant. The end plate was sutured to the sclera at a measured distance of 8-10 mm posterior to the limbus. Anterior chamber entry was made using a 23-gauge needle. A long oblique tunnel was created to prevent loss of fluid through the needle track. After the tube was inserted, the entry site was checked for leakage using a triangular cellulose sponge. In no case a leakage was observed. The limbal portion of the tube was covered with a pericardium patch.

\section{Postoperative IOP measurement and data analysis}

Intraocular pressure was measured postoperatively on the same day, $6 \mathrm{~h}$ after the surgery, using a Goldman applanation tonometer. The differences between pressures $\mathrm{A}$ and $\mathrm{B}, \mathrm{C}$ and $\mathrm{B}$, and $\mathrm{A}$ and $\mathrm{C}$ were calculated. Means and standard deviations were computed. Low IOP was defined as any value below $5 \mathrm{~mm} \mathrm{Hg}$. Pressure values of AGV with postoperatively low IOP were compared with those without low IOP using a two-sample $t$-test with equal variances.

Univariate analyses were used to assess each of the AGV pressure parameters and the differences against low IOP (as a dependent variable). Cut-off values for the two opening pressures and the closing pressure were calculated using the receiver operating characteristics (ROC) curve. The areas under the ROC curves (aROC) were calculated using the Stata 11.1 statistics software package (Stata Corp LP, College Station, TX, USA).

The probabilities for low IOP were estimated using a univariate logistic regression model, where a low postoperative eye pressure was entered as the dependent variate and valvular opening pressure was the independent variate. These values were plotted against the valvular opening pressure. To assess the overall reliability of the pressure measurement, we also calculated the correlation coefficients for the pairs of opening pressure, closing pressure, and re-opening pressure with the postoperative IOP.

\section{Results}

The clinical characteristics and the results of the opening and closing pressure measurements of the 30 patients are displayed in Table 1 . The first 10 patients are the cases with low postoperative IOP $<5 \mathrm{~mm} \mathrm{Hg}$.

Figure 1 presents all individual valve pressure parameters and the postoperative IOP. The opening pressure $\mathrm{A}$, closing pressure $\mathrm{B}$, and re-opening pressure $\mathrm{C}$ ranged from 5-30, 3-18, and 4-20 $\mathrm{mm} \mathrm{Hg}$, respectively. The means (SD) for these three pressure parameters were 18.4 (5.1), 8.3 (4.7), and $11.7(4.8) \mathrm{mm} \mathrm{Hg}$, respectively. The difference between the first opening pressure $\mathrm{A}$ and closing pressure B was 9.8 (4.8). The difference between the closing pressure $\mathrm{B}$ and the second opening pressure C was 3.3 (2.5).

Early after AGV implantation, 10 patients (33.3\%) had low IOP. Three patients in this group suffered from IOPrelated complications, such as shallow anterior chamber and choroidal effusion. Two cases (patient no 5 and 9) were managed successfully with injection of viscoelastics 


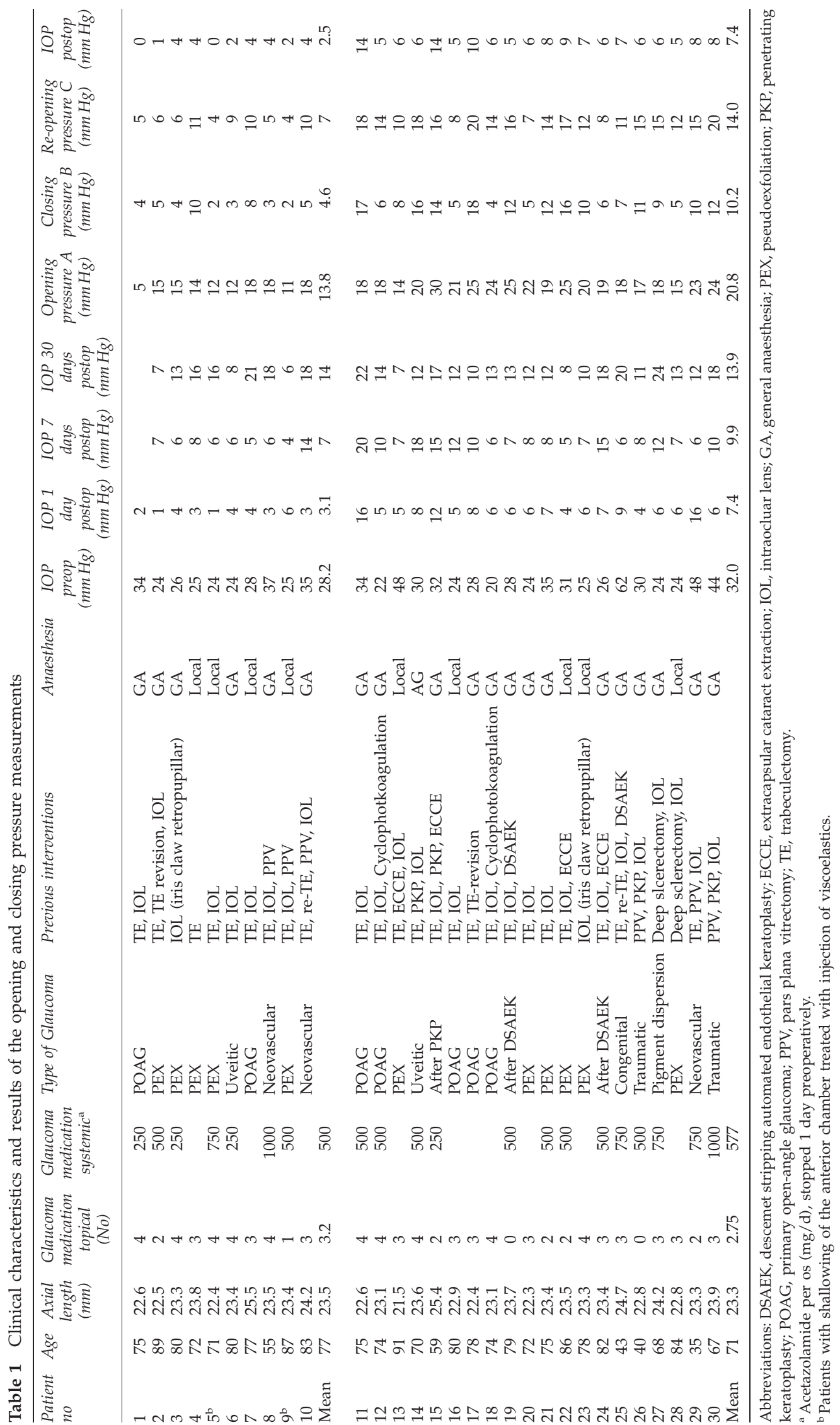


into the anterior chamber; in the 3rd case (patient no 1), the AGV was changed on the first postoperative day because of very shallow anterior chamber.

The 10 AGVs that resulted in low IOP showed significantly different mean opening pressure A, closing pressure $\mathrm{B}$, and re-opening pressure $\mathrm{C}$ compared with those measured in AGVs without low postoperative IOP (Table 2).

Low IOP was associated with opening pressure A (OR, 0.55; 95\% confidence interval (CI), 0.35-0.87; $P=0.010)$, re-opening pressure C (OR, 0.56; 95\% CI, $0.37-0.84 ; P=0.006)$, and to a lesser extent closing pressure B (OR, 0.61; CI, 0.41-0.9; $P=0.013)$; the corresponding aROCs were $0.91(0.81-1.00), 0.93$ (0.84-1.00), and 0.88 (0.76-1.00), respectively (Figure 2).

An opening pressure A value of $\leq 18 \mathrm{~mm} \mathrm{Hg}$ had a sensitivity (10/10) of $100 \%$ (95\% CI, 69.2-100) and a specificity $(13 / 20)$ of $65.0 \%$ (95\% CI, 40.8-84.6) for identifying AGV that resulted in low IOP. For the closing pressure $\mathrm{B}$, a cut-off value of $\leq 10 \mathrm{~mm} \mathrm{Hg}$ had a

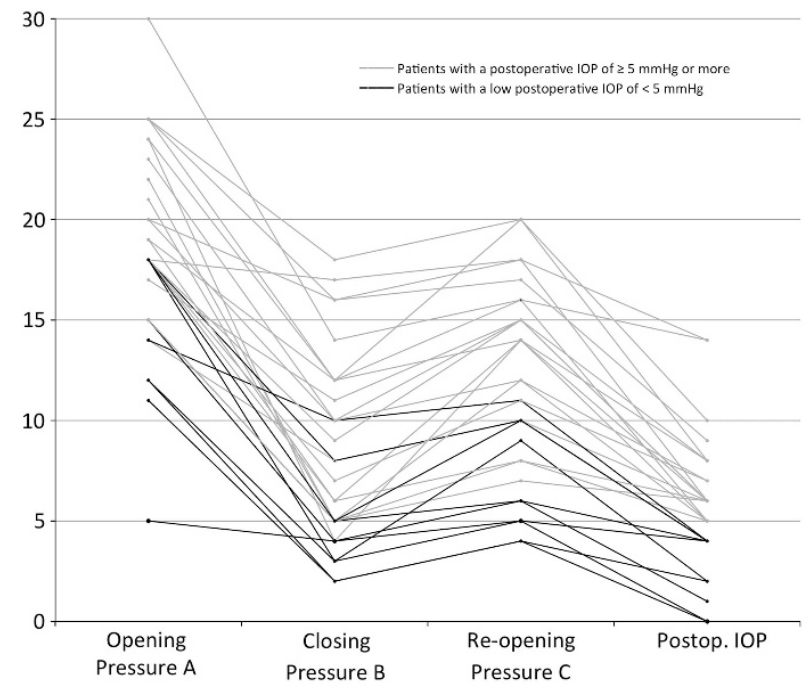

Figure 1 Opening pressure $\mathrm{A}$, closing pressure $\mathrm{B}$, re-opening pressure C, and postoperative intraocular pressure of each patient.

Table 2 Mean IOP values of the 10 AGVs that resulted in low postoperative IOP compared with the measured values for the 20 AGVs without low postoperative IOP

\begin{tabular}{lccc}
\hline & $\begin{array}{c}\text { AGV with low } \\
\text { postoperative IOP } \\
(\mathrm{n}=10)\end{array}$ & $\begin{array}{c}\text { AGV without low } \\
\text { postoperative IOP } \\
(\mathrm{n}=20)\end{array}$ & $\mathrm{P}$ \\
& & & \\
\hline Opening pressure A & $13.8 \mathrm{~mm} \mathrm{Hg}$ & $20.75 \mathrm{~mm} \mathrm{Hg}$ & 0.0001 \\
Closing pressure B & $4.6 \mathrm{~mm} \mathrm{Hg}$ & $10.15 \mathrm{~mm} \mathrm{Hg}$ & 0.0011 \\
Re-opening & $7.0 \mathrm{~mm} \mathrm{Hg}$ & $14.0 \mathrm{~mm} \mathrm{Hg}$ & 0.0001 \\
pressure C & & & \\
\hline
\end{tabular}

${ }^{\text {a }}$ Postoperative IOP $<5 \mathrm{~mm} \mathrm{Hg}$. sensitivity (10/10) of $100 \%$ (95\% CI, 69.2-100) and a specificity $(9 / 20)$ of $45.0 \%$ (95\% CI, 23.1-68.5). For the re-opening pressure $\mathrm{C}$, a cut-off value of $\leq 11 \mathrm{~mm} \mathrm{Hg}$ had a sensitivity (10/10) of $100 \%(69.2-100)$ and a specificity (15/20) of $75.0 \%$ (50.9-91.3).

The correlation coefficients between postoperative IOP and the three pressure parameters were 0.73 for opening pressure A, 0.78 for closing pressure B (Figure 3), and 0.75 for re-opening pressure $\mathrm{C}$.

\section{Discussion}

In the preoperative assessment of silicone AGV, we observed a large variability of opening, closing, and re-opening pressures. Re-opening pressure $\mathrm{C}$ with a cut-off value of $11 \mathrm{~mm} \mathrm{Hg}$ was the most helpful parameter in identifying all AGVs that resulted in low IOP, while closing pressure B showed the best correlation between measured pressure values and postoperative IOP.

We also observed substantial variations in the differences between the opening and closing pressures. Thus, even if the pressure level leading to activation of the AGV is known, it is not possible to predict the pressure value at which the valve will stop the drainage. Furthermore, the second activation of the valve immediately after it had closed required less pressure, indicating that the pressure range over which one single $\mathrm{AGV}$ is active is also variable.

These results have an impact on the clinical use of the $\mathrm{AGV}$, as they raise questions about the reliability of the valve mechanism as well as the priming procedure. A simple test of the specific AGV would be helpful to provide the surgeon with reliable information about its postoperative valve function. The herein described test procedure allows the prediction of situations with low postoperative IOP. In eyes that are prone to develop early postoperative hypotony, appropriate preventive measures can be taken, including exchange of the AGV before implantation.

It is important to note that low IOP does not automatically result in complications such as choroidal detachment or a shallow anterior chamber. Risk factors for a shallow anterior chamber after implantation of a glaucoma drainage device include myopia, small eyes, younger age, and fewer previous intraocular surgery. 5,6 In such cases, we recommend discarding the AGV if the opening pressure is $18.0 \mathrm{~mm} \mathrm{Hg}$ or less.

Our data correlate well with the relatively high rate of hypotony reported in literature after implantation of silicone AGV, ranging from 1.8-49\%.3,7,8 Another study reported hypotony-associated complications at rates of $19 \%$ (shallow anterior chamber) and 15\% (choroidal effusion). ${ }^{9}$ Interestingly, our results confirm the findings of a study where silicone AGVs were tested in vitro; 


\section{a}

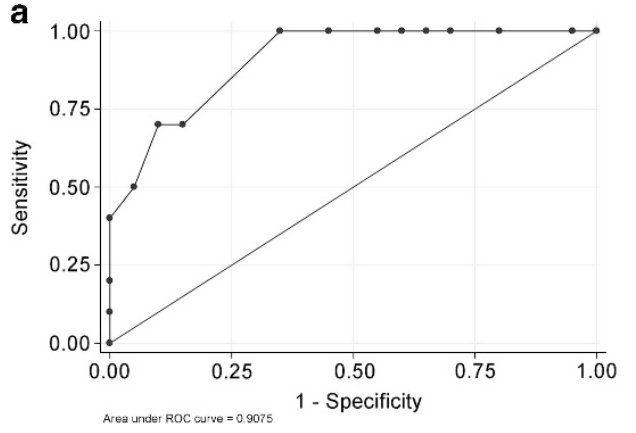

b

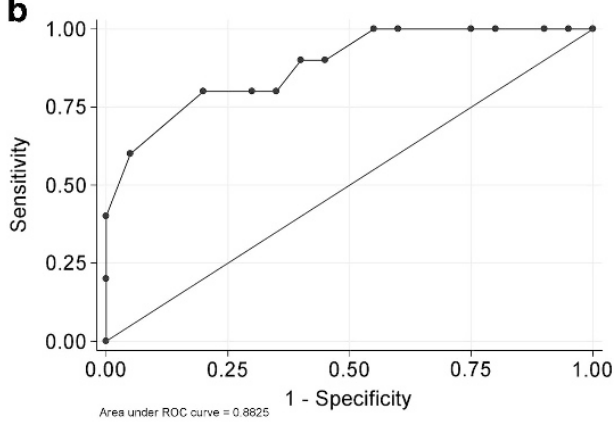

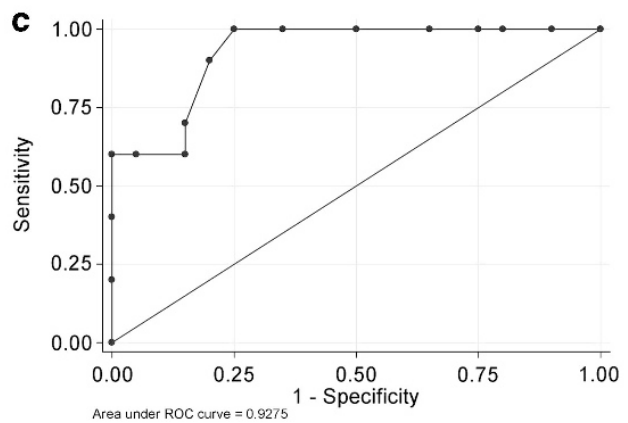

Figure 2 Receiver operating characteristic (ROC) curves for the opening pressure (a), closing pressure (b), and re-opening pressure (c).

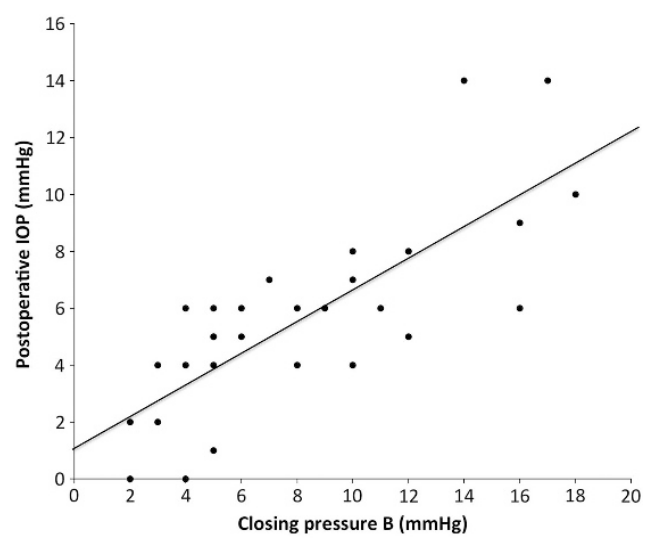

Figure 3 Correlation between closing pressure $B$ and postoperative IOP.

Moss and Trope ${ }^{4}$ reported that three out of six tested AGVs showed a very low closing pressure, ranging from 1.4 to $3.5 \mathrm{~mm} \mathrm{Hg}$.

Our study has several limitations. The test setting included subjective judging of the valve characteristics by the surgeon, and may have a limited accuracy. It is possible that loss of fluid along the tube track or low aqueous production could explain low postoperative IOP. However, the operation technique using a long oblique tunnel through which the tube enters the anterior chamber reduces the risk of leakage along the tube. Furthermore, it has been shown that flow characteristics might change in dynamic or steady-state flow conditions, ${ }^{10}$ and an in vitro study showed nonlinear resistance to flow through an AGV. ${ }^{11}$ Therefore, the test settings may not reflect in vivo conditions. However, the good correlation of the measured pressure parameters with the postoperative IOP indicates that the measurement is useful and reliable.

The reasons for the observed variability of the opening and closing pressures and for the highly variable pressure range over which an AGV is active are unclear. Therefore, the valve function as well the priming process of the AGV should be the focus of further research. It is possible that a standardised priming procedure could lead to more predictable valve function.

The valve function may not only play an important role in the early postoperative phase, but may also impact the long-term success of the glaucoma surgery. Like the surface structure and the shape of the $\mathrm{AGV}^{12}$ the valve function defining the amount of drained aqueous accessing the sub-Tenon tissue may also influence the wound-healing response and the formation of an inflammatory capsule around the AGV.

In conclusion, pre-implantation assessment of the valve function is recommended. Our results showed that each of the three pressure parameters could identify AGV's that resulted in low postoperative IOP with a cutoff value of $18 \mathrm{~mm} \mathrm{Hg}$ for the opening pressure, $10 \mathrm{~mm} \mathrm{Hg}$ for the closing pressure, and $11 \mathrm{~mm} \mathrm{Hg}$ for the 
re-opening pressure. These threshold values should trigger an intraoperative exchange of AGV in cases prone to hypotony.

\section{Summary}

What was known before

- Glaucoma drainage devices are increasingly used to treat refractory glaucoma.

- The Ahmed glaucoma valve (AGV) is equipped with a valve-like mechanism that should prevent early postoperative hyperfiltration and hypotony.

- Despite this outflow restriction, hypotony and hypotonyassociated complications are frequently reported in literature.

- In vitro studies have shown a high variability of the opening and closing pressures needed to activate or deactivate the AGV.

- This may explain cases of hypotony after AGV implantation.

What this study adds

- We present a reliable method to assess the valve function of an AGV intraoperatively.

- Just prior to the implantation, the opening, closing, and re-opening pressure were measured using a simple test set-up.

- In the preoperative assessment of silicone AGV, we observed a large variability of opening, closing, and reopening pressures.

- The closing pressure showed the best correlation between measured pressure values and postoperative IOP.

- Each of the measured pressure parameters could identify AGV's that resulted in low postoperative IOP.

\section{Conflict of interest}

The authors declare no conflict of interest.

\section{Authorship statement}

All listed authors contributed substantially to the manuscript and meet the criteria for authorship according to the ICMJE guidelines. In particular, all listed authors were involved in conception and design of the study, acquisition of data or analysis, and interpretation of data. All authors revised the manuscript critically for its intellectual content and approved the final version.

\section{References}

1 Schwartz KS, Lee RK, Gedde SJ. Glaucoma drainage implants: a critical comparison of types. Curr Opin Ophthalmol 2006; 17: 181-189.

2 Budenz DL, Barton K, Feuer WJ, Schiffman J, Costa VP, Godfrey DG et al. Ahmed Baerveldt Comparison Study Group. Treatment outcomes in the Ahmed Baerveldt Comparison Study after 1 year follow-up. Ophthalmology 2011; 118: 443-452.

3 Law SK, Nguyen A, Coleman AL, Caprioli J. Comparison of safety and efficacy between silicone and polypropylene Ahmed glaucoma valves in refractory glaucoma. Ophthalmology 2005; 112: 1514-1520.

4 Moss EB, Trope GE. Assessment of closing pressure in silicone Ahmed FP7 glaucoma valves. J Glaucoma 2008; 17(6): 489-493.

5 Park HY, Lee NY, Park CK. Risk factors of shallow anterior chamber other than hypotony after Ahmed glaucoma valve implant. J Glaucoma 2009; 18: 44-48.

6 Kee C. Prevention of early postoperative hypotony by partial ligation of silicone tube in Ahmed glaucoma valve implantation. J Glaucoma 2004; 13: 38-45.

7 Ishida K, Netland PA, Costa VP, Shiroma L, Khan B, Ahmed II. Comparison of polypropylene and silicone Ahmed glaucoma valves. Ophthalmology 2006; 113: 1320-1326.

8 Mackenzie PJ, Schertzer RM, Isbister CM. Comparison of silicone and polypropylene Ahmed glaucoma valves: twoyear follow up. Can J Ophthalmol 2007; 42: 227-232.

9 Barton K, Gedde SJ, Budenz DL, Feuer WJ, Schiffman J. Ahmed Baerveldt Comparison Study Group. The Ahmed Baerveldt Comparison Study methodology, baseline patient characteristics, and intraoperative complications. Ophthalmology 2011; 118: 435-442.

10 Francis BA, Cortes A, Chen J, Alvarado JA. Characteristics of glaucoma drainage implants during dynamic and steadystate flow conditions. Ophthalmology 1998; 105: 1708-1714.

11 Stay MS, Pan T. Thin-film coupled fluid-solid analysis of flow through the Ahmed Glaucoma Device. J Biomech Eng 2005; 127: 776-781.

12 Choritz L, Koynov K, Renieri G, Barton K, Pfeiffer N, Thieme H. Surface topographies of glaucoma drainage devices and their influence on human tenon fibroblast adhesion. Invest Ophthalmol Vis Sci 2010; 51: 4047-4053. 